Revue scientifique francophone en Communication

organisationnelle

$18 \mid 2000$

Non-verbal, communication, organisation

\title{
Non-verbal, communication, organisation - Exposé introductif
}

\section{Hugues Hotier}

\section{OpenEdition}

\section{Journals}

Édition électronique

URL : https://journals.openedition.org/communicationorganisation/2394

DOI : 10.4000/communicationorganisation.2394

ISSN : 1775-3546

\section{Éditeur}

Presses universitaires de Bordeaux

\section{Édition imprimée}

Date de publication : 1 novembre 2000

ISSN : 1168-5549

Référence électronique

Hugues Hotier, "Non-verbal, communication, organisation - Exposé introductif », Communication et organisation [En ligne], 18 | 2000, mis en ligne le 27 mars 2012, consulté le 05 août 2021. URL : http:// journals.openedition.org/communicationorganisation/2394; DOI : https://doi.org/10.4000/ communicationorganisation.2394

Ce document a été généré automatiquement le 5 août 2021.

(C) Presses universitaires de Bordeaux 


\title{
Non-verbal, communication, organisation - Exposé introductif
}

\author{
Hugues Hotier
}

1 L'organisateur de ce colloque est le GREC/O, Groupe de Recherche en Communication des Organisations. Cependant, chacun sent bien que ce dont il sera ici question ne ressortit pas exclusivement à la communication des organisations. A qui chercherait à comprendre comment un groupe que son intitulé voue à l'étude de la communication organisationnelle peut ainsi élargir son champ, sinon le quitter, il faudrait répondre qu'il s'agit d'une impérieuse obligation. Nous n'avons pas opté pour une approche à la fois plus large et plus fondamentale de la communication. Nous y avons été contraints.

2 Fondé en janvier 1987, notre laboratoire s'appela d'abord GREC. Groupe de Recherche Entreprise et Communication. Sans doute croyions-nous à cette époque que l'entreprise était un objet de recherche suffisamment important pour lui consacrer l'intégralité de nos préoccupations. Assez vite, nous nous rendîmes compte que la notion d'entreprise était trop restreinte et qu'il fallait l'inscrire dans une catégorie supérieure, celle des organisations. Ce qui peut sembler banal aujourd'hui était moins évident il y a une douzaine d'années. Nous changeâmes de nom et d'objet sensiblement au moment où des sous-disciplines tentaient de s'imposer au sein de ces sciences aussi floues que molles qu'on appelait - et qu'on appelle toujours, je me demande si l'imparfait n'est pas un lapsus - Sciences de l'Information et de la Communication. Depuis une trentaine d'années qu'on parle de sciences de l'information et de la communication, le débat ne s'est pas apaisé malgré les artifices lexicaux auxquels on recourt pour en faire une transdicipline ou une interdiscipline. Périodiquement resurgit l'insupportable question: pourquoi parle-t-on de sciences au pluriel et non d'une communicologie comme il existe une sociologie ou une ethnologie ? Est-on en présence d'une discipline ou bien d'une juxtaposition de disciplines qui se pencheraient sur un même objet? En d'autres termes, la communication est-elle un objet si particulier qu'il justifierait une science spécifique? Bien d'autres questions se posent encore qui agitent les conseils, comités et autres cénacles. Par exemple, le récurrent problème de la réunion en une même discipline de l'information et de la communication. 
On s'est suffisamment disputé en d'autres lieux pour que nous n'abordions pas ici ces dangereux sujets de discorde. Nous les laisserons de côté pendant ces deux journées, heureux de constater qu'ils n'ont pas empêché le GREC/O de fonctionner, de produire et de vivre en bonne harmonie pendant treize années. Mais nous ne pouvons passer sous silence une évidence qui s'est imposée à nous depuis quelques années. Je ne sais pas si les sociologues font leur cette distinction communément admise qui parle d'une sociologie du travail, d'une sociologie des médias, d'une sociologie des religions, d'une sociologie des organisations... Pour nous, il me semble que nous sommes de moins en moins convaincus, à tout le moins au sein du GREC/O, de l'existence d'une communication des organisations. Pas plus d'ailleurs que d'une communication sociale ou d'une communication publicitaire. Ce qui s'impose à nous, de manière de plus en plus prégnante me semble-t-il, c'est l'existence de réalités transversales qui dépassent et englobent ces classifications. Le non-verbal, qui ne s'oppose pas au verbal, est au nombre de ces réalités. Ce que nous avons, il y a trois ans lors de notre précédent colloque, appelé induction aussi.

Il me faut ici introduire une indispensable précision à propos de la communication des organisations et de l'apparente distance que nous prenons par rapport à celle-ci. Nous ne refusons pas d'étudier la communication des organisations. Nous commençons à penser que. vraisemblablement, elle n'est pas une communication spécifique. Et le détour que nous faisons par le fondamental et le théorique, par la compréhension des réalités transversales, ne peut que nous aider à approcher et traiter la forme particulière que revêt la communication lorsqu'elle s'observe au sein des organisations.

5 En 1997, nous avions cru mettre un point final à un programme de recherche qui nous avait tenu en haleine pendant plus de trois années en consacrant notre dixième colloque à «Induction et communication ». Chercheurs français et étrangers prirent part à un passionnant débat qui apparut vite comme une invitation à repartir sur ces chemins peu explorés. Repéré comme un des moyens privilégiés de l'induction, le nonverbal devint l'objet central de la réflexion du GREC/O, laquelle réflexion a donné naissance à un ouvrage qu'on peut considérer comme le point de départ de notre réunion d'aujourd'hui ${ }^{1}$

6 L'usage voudrait que, introduisant ce colloque, je propose une définition du non-verbal, fût-elle provisoire, afin que nous nous mettions d'accord au moins sur le lexique. Ainsi avions-nous procédé lors de notre précédent colloque en proposant du mot induction une définition que nous avions élaborée après de longues heures de discussion au sein de notre groupe de recherche. Ce n'est pas ainsi que nous avons agi cette fois : nous n'en avons pas éprouvé le besoin, comme si tout le monde était d'accord sur la définition du non-verbal. Cependant, nous avons avec prudence et, j'espère, sagesse, organisé une table ronde pour ouvrir les débats. Nous avons chargé un sociologue, un sémioticien et un éthologue de la communication de nous éclairer en se penchant sur la polysémie du mot non-verbal.

7 Peut-être penserez-vous que c'est là une façon de transférer les responsabilités. Peutêtre. Alors, continuant dans cette voie, j'aimerais, pour ce qui me concerne, me référer au livre de Jacques Corraze, Les communications non-verbales, publié en 1980. Vous voyez que, loin de proposer notre définition, a fortiori ma définition, j'entends me réfugier derrière l'autorité d'un collègue que je soumets à votre réflexion sinon à votre critique. Pour que ma démarche ne vous paraisse pas tout à fait malhonnête, je m'empresse de dire que sa manière de poser le problème me convient fort bien et qu'il 
ne me semble pas nécessaire, dans cette introduction, de disserter davantage. Je ne doute pas que nos débats nous permettront d'aller plus loin, de conclure plus richement, mais le moment n'en est pas encore venu.

8 Voici donc les éléments de définition que Jacques Corraze livre dès la première partie du chapitre premier :

9 «On entend par communications non-verbales l'ensemble des moyens de communication existant entre des individus vivants $n$ 'usant pas du langage humain ou de ses dérivés non sonores (écrits, langage des sourds-muets, etc). La définition commence par une exclusion. [...]

10 Le verbe $n$ 'est évidemment pas ici la catégorie grammaticale, ni le canal vocal. Une communication non-verbale peut être sonore et il faut se garder de l'expression «langage silencieux» pour qualifier les communications non-verbales. Ce que ce concept exclut c 'est le système linguistique humain ; c 'est lui qui est verbal. [...]

11 On applique le terme de communications non-verbales à des gestes, à des postures, à des orientations du corps, à des singularités somatiques, naturelles ou artificielles, voire à des organisations d'objets, à des rapports de distance entre les individus, grâce auxquels une information est émise. » (pp. 12-13)

12 Après de nombreux auteurs, dont Dittmann qu'il cite ("Kinesics and context ", in Psychiatry, 1971), Corraze fait observer que l'homme maîtrise très peu ses communications non-verbales, voire y accorde peu d'attention, sauf dans des situations particulières; par exemple, dans les cas de simulation (au sens de tromperie). Cependant, le contrôle que l'homme exerce, probablement inconsciemment, sur son expression non-verbale résulte le plus souvent d'une éducation qui transmet les interdits culturels en matière d'expression publique des émotions.

13 Corraze fait sienne la définition du psychanalyste Scheflen («The signifiance of postures in communication Systems », in Psychiatry, 1964) : «La communication... comprend tous les comportements par lesquels un groupe constitue, soutient, médiatise, corrige et intègre ses relations ». Ce faisant, il récuse les grilles de lecture qui permettent d'interpréter un peu facilement gestes et postures dans une démarche modélisante. Il critique l'ouvrage de Feldman. Mannierisms of speech and gesture in everyduy life (Indiana University Press, 1959) dans lequel « Nous apprenons ainsi que le clignement des yeux, chez l'enfant, représente le désir de dissimuler la masturbation et, chez les femmes, celui de découvrir ses organes génitaux. » (p. 21).

14 Les cadres et les chefs d'entreprise qui s'inscrivent aux séminaires de la programmation neuro-linguistique n'en demandent pas plus : la modélisation paraît être le nouveau prêt à penser, en tout cas le raccourci le plus efficace vers l'opérationnalité. l'instrumentalisation. de pseudo-sciences aux prétentions artistiques ainsi que l'écrivent Joseph O'Connor et John Seymour dans leur Introduction à la PNL :

15 «La PNL est l'art et la science de l'excellence personnelle. C 'est un art en cela que chacun imprime à tout ce qu'il fait la personnalité et le style qui lui sont propres, et qui ne peuvent jamais être figés dans des mots ou des techniques. C 'est une science, parce qu'il existe un processus de définition des modèles appliqués par les individus remarquables dans leur domaine pour atteindre leurs résultats exceptionnels. Il s'agit de la modélisation; les modèles, les tours et les techniques découverts sont de plus en plus utilisés dans les activités de conseil, d'enseignement et dans les affaires pour parvenir à une communication plus efficace, au développement personnel et à un 
apprentissage accéléré. [...] La PNL est une technique qui procure les résultats auxquels nous aspirons, tout en mettant les autres en valeur. C 'est l'étude de ce qui différencie l'excellence de la moyenne. Elle apporte aussi un corpus de techniques extrêmement efficaces applicables à l'enseignement, au conseil, aux affaires et à la thérapie.» (Editions Vigot, 1995, pp. 17-18)

16 Pour compléter l'approche de Jacques Corraze, j'aimerais me référer à Claude Hagège afin d'évoquer une des questions que ne manquera pas de traiter ce colloque, si j'en juge par les contributions qui nous ont été soumises : celle des insuffisances de l'écrit par rapport à l'oral ou, pour dire autrement les choses, des frustrations que provoque le passage de l'oral à l'écrit, quel qu'en soit d'ailleurs le support, papier ou écran. Dans L'Homme de paroles (Fayard, 1985, pp. 83-85), Claude Hagège écrit :

«La communication orale, seule naturelle, est seule chargée de tout le sens d'origine. Elle est multiplanaire. Un phénomène capital, dont aucun système d'écriture connu ne conserve la trace, le fait bien apparaître. Ce phénomène est l'intonation. Dans l'Antiquité, les grammairiens et certains philosophes avaient fort bien vu que les textes latins, par exemple, peuvent, faute de noter les courbes intonatives, aboutir à des contresens (comme celui que l'on commet lorsque l'on prend une interrogation pour une assertion), ou à des absurdités. [...] L'intonation stratifié souvent le discours oral en une structure hiérarchique où le message principal n 'est pas prononcé sur le même registre que les incises, éventuellement imbriquées les unes dans les autres. [...]

18 Ainsi, pour essentielle qu'elle soit dans le destin des hommes, ou de la partie d'entre eux qu'elle concerne, l'invention de l'écriture a contribué à occulter l'exercice vivant de la parole. Les pictogrammes, les idéogrammes, les phonogrammes, les syllabaires, les alphabets demeurent des projections graphiques défuntes, et insuffisantes, de la gestuelle articulatoire et des sémiotiques expressives comme celle du visage. [...]

19 Le style oral, quant à lui. recourt à toutes sortes de procédés de symbolique gestuelle et articulatoire qui lui assurent une étonnante efficacité mnémotechnique: refrains, syllabes de déclenchement, mots d'appel, noms-agrafes, expressions inductrices, profusion de quasi-synonymes, assonances, rimes, allitérations et autres échos phoniques et sémantiques, parallélismes lexicaux et grammaticaux, couples de sens, rythmisation par le geste et par les mouvements de la bouche."

Il pose là le problème important de l'insuffisance de la communication symbolique, à moins qu'il faille dire culturelle, par rapport à la communication naturelle. Il y a là un réel problème de traduction auquel la ponctuation et la typographie ont tenté d'apporter des solutions. On sait que l'écriture sur écran cherche ses propres solutions en chargeant de petites icônes de transmettre l'intention qu'on trouve souvent, à l'oral, dans l'intonation.

21 Quand des chercheurs invitent d'autres chercheurs à débattre en leur soumettant comme point de départ les résultats de leurs propres travaux, ils établissent avec leurs invités un contrat implicite fondé sur la productivité du colloque. Pour dire les choses différemment, ils s'engagent à organiser le colloque de telle sorte que chacun des participants puisse à la fois en tirer le plus possible d'enseignements et s'ouvrir de nouvelles pistes de réflexion. Il ne s'agit évidemment pas de terminer par un triomphal C.Q.F.D. mais de relancer la recherche, de lui ouvrir de nouvelles avenues. Ce projet, classique et ambitieux, nécessite une organisation, une mise en forme des débats, une discipline aussi. 
Le premier écueil, la première décision qui vous vaudra reproche, concerne la structuration du colloque. Thèmes, sous-thèmes, parties? Comment organiser la présentation des contributions? Quelle économie imposer à ces propositions qui nous sont soumises? Fort heureusement, la préparation d'un tel colloque est un travail collectif. La vingtaine de chercheurs qui composent le GREC/O a longuement réfléchi pour, finalement retenir trois entrées. Chacune est accompagnée d'une définition qui, comme toutes les définitions, est sans doute contestable, mais qui a le mérite d'exprimer ce que nous entendons par les dénominations de ces entrées, comment nous considérions, au moment où nous les avons formulés, ces choix dont la portée est d'ailleurs susceptible d'être remise en question par nos débats.

La relation duelle

La relation duelle, provoquée ou fortuite, comporte une dimension non-verbale souvent fondamentale dans l'activation des processus de communication, médiatisée ou non.

\section{Le collectif}

La règle des trois unités définit parfaitement le collectif au sens où nous l'entendons : un rassemblement temporaire en un lieu, autour d'un événement. Le non-verbal peut être constitutif de l'événement à l'origine du rassemblement comme il peut s'observer dans la dynamique collective.

L'organisation

Les organisations sont fondées sur des processus rationnels. L'organisation de ces processus passe par le langage. Cependant, force est de constater la présence du nonverbal dans cet édifice de rationalité. Comment et pourquoi se manifeste-t-il ? Avec quels effets dans les organisations modernes?

29 On comprend qu'il ne s'agit pas là de trois sous-thèmes mais, pour reprendre une expression que nous avions employée lors du colloque de 1997, de trois vues en coupe d'un même phénomène.

30 Pour chacune de ces entrées, on donnera d'abord la parole à un conférencier avant que le président de séance n'introduise les contributions retenues par le comité scientifique, lesquelles seront suivies d'un débat avec le public. Le conférencier, ou une autre personnalité en cas de dédoublement en ateliers, terminera en livrant ses impressions à chaud après l'audition des communications et du débat. Il ne s'agit pas de synthétiser ce qui a précédé mais bien d'épiloguer. C'est pourquoi nous parlons de " tireur d'épilogue »²

1 La quantité des propositions que nous avons reçues, et la grande qualité de nombre d'entre elles, nous ont amenés à organiser des ateliers simultanés. C'est une pratique nouvelle que nous inaugurons avec ce 11èmecolloque du GREC/O. Nous espérons que les participants ne seront pas trop frustrés face à des choix souvent difficiles.

J'ai employé le mot discipline qui peut étonner ceux qui ne sont pas des habitués de nos colloques. L'existence d'ateliers concomitants nous incite davantage encore que dans les colloques précédents à nous arrêter un instant sur cette caractéristique des assemblées du GREC/O. Nous avons, certes par écrit avec ce que cela comporte d'insuffisances, mais souvent aussi oralement avec la conviction que confèrent l'intonation, la mimique et la gestuelle, indiqué et confirmé aux intervenants que l'exposé de leur contribution ne saurait en aucun cas excéder les 20 minutes imparties. 
En m'imposant la plus grande sobriété gestuelle et mimique, je voudrais confirmer à nouveau que cette règle est impérative. Il ne s'agit pas pour nous d'exercer un quelconque arbitraire que nous permettrait le pouvoir de l'organisation mais bien de garantir au dernier intervenant d'un atelier que ses prédécesseurs n'auront entamé ni son temps ni la disponibilité de ses auditeurs et de faire en sorte que les débats qui suivront les exposés aient une durée suffisante pour que d'authentiques échanges puissent avoir lieu. Pour éviter aux modérateurs la fastidieuse rédaction de petits papiers qu'on fait passer aux communicateurs agacés, nous avons imaginé une procédure simple inspirée du football et, plus précisément, de la finale de la Coupe de France que nous avons tous regardée pour des raisons strictement épistémologiques. Dans chaque atelier nous avons installé un arbitre assistant, un juge de touche en quelque sorte, pour seconder l'arbitre modérateur. C'est lui qui indiquera d'un carton jaune que nous sommes à 2 minutes de la fin de l'exposé. Quand, à la dernière seconde, il brandira le carton rouge, le communicateur bavard devra, même s'il est sur le point de marquer un but décisif, interrompre sa phase de jeu et quitter le terrain sans discuter s'il veut éviter la honte du sifflet ${ }^{3}$.

Il me reste à souhaiter que ces deux journées soient profitables et agréables à tous. Je crois qu'elles seront profitables parce que nous attendons tous beaucoup de ce colloque pour enrichir notre propre réflexion et que nous sommes prêts, les uns et les autres, à confronter nos connaissances et nos idées à cette fin. Je sais qu'elles seront agréables parce que les étudiants du DESS en Information et Communication des Organisations qui ont pris en main la partie logistique de la manifestation, avec l'équipe administrative du GREC/O - Fabienne Arcos, Marie Navarro et Aline Simon-Lalande ont tout fait pour qu'il en soit ainsi. Je voudrais, d'ores et déjà, les en remercier.

\section{NOTES}

1. non verbal et organisation, Paris, L'Harmattan, 2000

2. Tireur d'épilogue n'est pas, à proprement parler, un nouveau métier et, sauf évolution significative du marché de l'emploi, nous n'envisageons pas d'ouvrir un DESS spécifique. Cependant, force est de constater qu'il s'agit là d'une compétence pouvant entrer dans un profil de poste. Sans prétendre avoir inventé la chose, le GREC/O se félicite d'avoir trouvé le mot et de contribuer ainsi à donner ses lettres de noblesse à cette fonction des plus utiles.

3. Le Deuxième séminaire interculturel sino-français de Canton, qui s'est tenu du 9 au 11 juin à l'Université des Etudes Etrangères du Guangdong, a adopté notre système. Le sens de la face, qui est un des fondements de la culture chinoise et qu'Erving Goffman a si bien analysé, a entraîné une amélioration de la procédure du GREC/O : ronds plutôt que rectangulaires, les cartons portaient les traits caractéristiques d'un visage souriant afin que la discipline imposée soit exempte de méchanceté. Voilà un exemple remarquable de transfert de technologie. 